\title{
A Systematic Narrative Review of Cognitive-Behavioral Therapies with Asian American Youth
}

\author{
Rongyu Xin ${ }^{1}$, Olivia M. Fitzpatrick ${ }^{2}$, Patrick Ho Lam Lai ${ }^{3}$, John R. Weisz ${ }^{2}$, and Maggi A. \\ Price $^{2,3}$ \\ ${ }^{1}$ Graduate College of Education, Lehigh University \\ ${ }^{2}$ Department of Psychology, Harvard University \\ ${ }^{3}$ School of Social Work, Boston College
}

\begin{abstract}
Author Note
Rongyu Xin ${ }^{D}$ https://orcid.org/0000-0003-0187-7472

Olivia M. Fitzpatrick $@$ https://orcid.org/0000-0001-8868-6702

Patrick Ho Lam Lai $\$ https://orcid.org/0000-0003-1904-265X

John R. Weisz $@$ https://orcid.org/0000-0003-0820-2347

Maggi A. Price $D$ https://orcid.org/0000-0001-9825-6925

We have no conflicts of interests to disclose.

Correspondence concerning this article should be addressed to Maggi A. Price, School of Social Work, Mcguinn Hall, Office 126, 140 Commonwealth Ave., Chestnut Hill, MA,
\end{abstract} 02467. Email: maggi.price@bc.edu Draft Version 3 (9/6/2021). Please do not copy or cite without the authors' permission. The preregistration is currently under review. 


\begin{abstract}
Asian American (AsA) youth comprise a large and fast-growing proportion of the U. S. population. AsA youth have comparable and sometimes higher rates of mental health concerns compared to White youth, but are significantly less likely to access mental health services. Cognitive-behavioral therapy (CBT) was originally designed by and for White and Western populations, but might provide clinical benefits for AsA youth because several CBT characteristics overlap with some AsA values (e.g., directive therapeutic style; family involvement). Despite this promise, there has yet to be a synthesis of evidence on the effectiveness of CBT, either culturally-adapted or non-adapted, for AsA youth. A systematic narrative review identified randomized controlled trials (RCTs) of CBT conducted with AsA youth. Electronic databases used included PsycINFO, Web of Science, PubMed, and ProQuest. Our search yielded 2,059 articles, of which, 8 RCTs (386 participants) met inclusion criteria. Studies were heterogeneous across targeted problems (e.g., phobia, depression) and age (M=8.422.1 years). Findings suggest that both culturally-adapted and non-adapted CBT (3 and 5 RCTs, respectively) were effective in reducing a range of emotional and behavioral problems for AsA youth. The dearth of studies with AsA youth underscores the need for enhancing the cultural responsiveness of clinical research and practice, as well as harnessing community-engaged methods to improve the accessibility and uptake of evidence-based mental health services for AsA youth.
\end{abstract}

Keywords: children, adolescents, psychotherapy, evidence-based practice, Asian Americans, cognitive-behavioral therapy, culturally adapted therapy 


\section{A Systematic Narrative Review of Cognitive-Behavioral Therapies with Asian American}

\section{Youth}

Asian Americans (AsA) comprise a large and fast-growing proportion of the United States (U.S.) population, growing by $72 \%$ in the last 20 years (López et al., 2017). AsA represent 19 country-of-origin groups (e.g., Filipino, Chinese) that vary across a multitude of characteristics both within and between groups, such as educational attainment (Budiman et al., 2019), economic privilege (Budiman et al., 2019), and cultural values (Heras, 2007; Kim et al., 2001). Despite the heterogeneity within AsA, this population has higher educational achievement

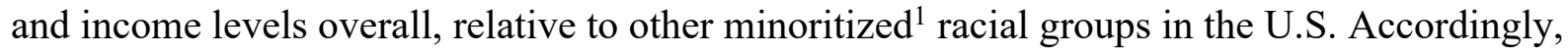
AsA are sometimes deemed the "model minority" (i.e., they are idealized and believed to work hard, behave well, and succeed compared to other minoritized racial and immigrant groups; Choi \& Lahey, 2006; Qin et al., 2008) and perceived as well-adjusted (Zhou et al., 2009). In addition to facing this and other harmful stereotypes, AsA experience significant and rapidly increasing discrimination and hate crime (McMurtry et al., 2019; Wu et al., 2020) that negatively impact their physical (Gee et al., 2007) and mental (Lee \& Ahn, 2011) health.

Despite the urgent need to understand AsA mental health and mental health care needs, AsA remain the most understudied racial group in psychology (Uba, 2003). Consistent with the model minority myth, mental health research often emphasizes academic achievement over mental health, especially for AsA children, adolescents, and young adults ("youth" herein; Qin et al., 2008). Accumulating evidence, however, suggests that AsA youth face numerous

\footnotetext{
${ }^{1}$ Minoritization refers to being placed into a "minority" status reflecting historical and
} structural marginalization, rather than statistical underrepresentation (Sotto-Santiago, 2019). 
psychological challenges. For instance, relative to White youth, AsA youth have comparable rates of eating disorders (Cheng et al., 2019) and are more likely to report depression (Song et al., 2011; Young, 2010). AsA youth may face different, and sometimes more severe, stressors compared to AsA adults, such as pressures to acculturate more quickly, conflicts between American culture and their family's culture (Lee et al., 2009), racially-motivated bullying and discrimination by peers (Ling et al., 2014) and teachers (Wang \& Atwal, 2015), and stress associated with acting as a language broker (i.e., translating and interpreting language for others, such as parents; Hua \& Costigan, 2012).

Although AsA youth may need mental health care to address these concerns, they face an array of cultural and structural barriers hindering their ability to identify these concerns, and access and utilize relevant services. Although a thorough review of these barriers is beyond the scope of the current paper, we provide a few examples. Culturally-specific attributions for mental health problems among AsA may include a lack of differentiation between mental and physical problems, and some AsA believe that problems can be mediated by willpower (Zhou et al., 2009). These beliefs may reduce the likelihood of seeking help from mental health professionals (Leong \& Lau, 2001).

At the structural level, AsA youth are less likely to be referred to school-based mental health services relative to other youth of Color (e.g., Latinx youth; Guo et al., 2014). It is possible that the model minority myth undergirds this disparity by encouraging teachers or other treatment-referring adults to view AsA youth as high-achieving students who do not require intervention. Experts suggest that an additional byproduct of this myth is stigma related to mental health challenges, or the notion that help-seeking reflects weakness (Chiang et al., 2021). 
Indeed, AsA youth are more likely than youth of other races to hide or deny their mental health problems, and less likely to seek professional help (Lee et al., 2009).

Due in part to the lack of language-appropriate mental health providers, AsA immigrants are less likely to access services relative to U.S.-born AsA (e.g., due to lower English proficiency; Abe-Kim et al., 2007; Le Meyer et al., 2009). Ongoing healthcare inequities (e.g., microaggressions $^{2}$, denial or minimization of concerns expressed by people of Color) appear to have contributed to understandable mistrust and in some cases fear of healthcare institutions among minoritized groups, including AsA (e.g., David, 2010; Jaiswal \& Halkitis, 2019; Sentell et al., 2007). Specifically, experiences of being stereotyped by providers are associated with AsA's underutilization of mental health services (Misra et al., 2021). Finally, AsA access to mental health services is hindered by practical barriers maintained by inequitable distribution of existing services and resources, including child care and transportation (Wang, Do, et al., 2019; Zhou et al., 2009).

Researchers have begun examining barriers to AsA youth's mental health treatment in recent years. This is evidenced by (1) the development and study of culturally-adapted treatments (i.e., interventions tailored for minoritized groups) for AsA (Huey \& Tilley, 2018) and (2) trials examining the effectiveness of existing non-adapted therapies with AsA (Chu \& Harrison, 2007; Lin, 2002; Tarrier et al., 2008). Notably, meta-analyses found that both culturally-adapted and non-adapted mental health treatments were effective for Chinese people (Ng \& Wong, 2018) and AsA (Huey \& Tilley, 2018), though adapted treatments were more effective in both studies.

\footnotetext{
${ }^{2}$ A microaggression refers to subtle, everyday communications that convey negative or denigrating racial insults toward minoritized groups (Sue et al., 2007).
} 
Although culturally-adapted treatments evidence promise, skeptics note that an infeasible number of studies are needed to design and test interventions for each cultural group, and each study necessitates considerable time and resources (O’Donohue \& Benuto, 2010). Given the relative infancy of research on culturally-adapted treatments for AsA and the growing need for AsA to access mental health services, it is important to understand the utility of existing treatments for this population. In particular, we sought to explore the potential utility of cognitive-behavioral therapy (CBT) - a broad psychotherapy approach involving cognitive (e.g., cognitive restructuring) and/or behavioral techniques (e.g., behavioral activation) to change unhelpful thinking patterns and/or maladaptive behaviors (Beck et al., 1979) - for AsA youth. We focused on CBT because some of its core features overlap with AsA cultural values, which may suggest that non-adapted interventions can effectively treat mental health problems among AsA (Hsieh \& Bean, 2014; Li \& Kim, 2004; Sandil, 2006).

\section{The Current Review}

The current systematic narrative review synthesizes evidence from randomized controlled trials (RCTs) testing CBT, either culturally-adapted or not, with AsA youth. This review is important for several reasons: (1) Most existing literature provides frameworks for intervention development or adaptation for AsA (e.g., Chen \& Davenport, 2005; Hsieh \& Bean, 2014; Leong, 1986), but few studies examine their efficacy (Huey \& Tilley, 2018). (2) It is critical to examine AsA youth specifically because they face different life stressors compared to AsA adults (e.g., racially motivated bullying) that may be differentially implicated in mental health risk or treatment needs (Ling et al., 2014). (3) Existing reviews of psychotherapy efficacy for AsA focus on specific ethnic subgroups (e.g., Chinese people; Ng \& Wong, 2018) and/or include a wide range of mental health interventions (e.g., Huey \& Tilley, 2018), while the current narrative 
review focuses on a specific modality that may be most appropriate for this population (e.g., CBT; Lau et al., 2011; Marchand et al., 2010). To more clearly understand the potential effectiveness of CBT for AsA youth, we first briefly describe the overlapping features of CBT and AsA cultural values. Next, we synthesize evidence from RCTs on CBT, either culturallyadapted or not, based on a systematic search. Finally, we closely examine these RCTs, summarize their findings, and discuss gaps in existing research and recommend future directions.

\section{Cognitive Behavioral Therapy and Asian American Values}

Experts have highlighted the overlap between core features of CBT and some common values shared across Asian cultures, suggesting that CBT may be appropriate for AsA (Chen \& Davenport, 2005; Iwamasa et al., 2019). It is important to note, however, that there is significant heterogeneity in Asian values endorsed within and across AsA subgroups (Kim et al., 2001). For instance, East AsA (e.g., Japanese, Koreans) may be more likely to endorse values of collectivism and filial piety (i.e., a virtue reflecting voluntary respect for one's parents) compared to Southeast AsA (e.g., Filipinos). Recognizing the complexity of AsA values, we summarize four rather broadly applicable core AsA values that appear to overlap with CBT below.

\section{Emphasis on Secondary Control}

Secondary control refers to changing one's subjective experience of a stressor (e.g., thoughts, emotions, attributions) instead of the actual conditions of that stressor (e.g., environment; i.e., primary control) and is considered to be fundamental to Asian culture (Rothbaum et al., 1982; McCarty et al., 1999; Zhou et al., 2009). For instance, scholars have posited that while Western cultures favor primary control, Chinese and Japanese people tend to possess an external locus of control, believing that their own actions have minimal influence on 
environmental factors (Lin, 2002; McCarty et al., 1999). Cognitive restructuring, a commonly used CBT technique designed to help clients modify their thinking patterns to be more adaptive (Beck et al., 1979), may align with the secondary control value of AsA.

\section{Primacy of Cognitions}

CBT often begins by focusing on cognitions (e.g., their identification and modification), and later addresses emotions - though often only as they relate to cognitions (Chen \& Davenport, 2005). In other words, cognitions are considered the primary driver of distress, and thus the primary lever to be adjusted to change associated emotions and behaviors (Beck et al., 1979). This intervention pattern and conceptual model may be particularly acceptable for AsA, as they tend to prefer focusing on cognitions over emotions. For example, East AsA avoid sharing intense emotions outside their family (Hynes, 2019) and may feel more comfortable sharing emotions after the therapeutic alliance has been established (Chen \& Davenport, 2005).

\section{Structured and Directive Approach}

Core features of CBT include its structured, and often manualized, nature (Hall et al., 2019) and the directive approach of CBT therapists (e.g., providing psychoeducation, assigning homework; Hwang et al., 2006). Scholars suggest that structured interventions may be more acceptable and engaging for AsA youth (Sandil, 2006) and that AsA might be especially inclined to complete between-session assignments (Iwamasa et al., 2019; Sandil, 2006). Given that AsA typically exhibit respect for authority figures and value interpersonal harmony (Hynes, 2019), therapeutic relationships wherein clinicians demonstrate expertise, explicitly suggest skills, and educate clients might be particularly desirable for AsA (Chen \& Davenport, 2005). In fact, several studies demonstrate that AsA prefer directive (vs. nondirective) approaches (Atkinson et al., 1978; Exum \& Lau, 1988; Li \& Kim, 2004). 


\section{Family Involvement}

CBT for youth often emphasizes family member involvement (e.g., parent-training; keeping parents informed about what their child is learning in therapy; Khanna \& Kendall, 2009). Family - often ranging from the nuclear to the extended family in AsA culture (Iwamasa et al., 2019) - is generally very important to AsA, and it is not uncommon for family members to regularly accompany AsA clients to therapy (Hwang et al., 2006). Given the cultural emphasis on AsA family interdependence (Kim et al., 2001), family involvement, which has been shown to enhance youth psychotherapy efficacy in general (Haine-Schlagel \& Walsh, 2015), might also increase treatment engagement for AsA youth (Sandil, 2006). Consistent with this possibility, qualitative evidence suggests that improving family cohesion during treatment is beneficial for the behavioral health of AsA youth (e.g., Filipino youth; Javier et al., 2018).

\section{Potential Limitations of CBT for AsA}

Despite the potential advantages and overlapping characteristics between CBT and AsA culture, it is also important to note some limitations of these interventions. First, CBT was originally created by and for White people in Western countries and is rooted in Western values (e.g., individualism; Benish et al., 2011). Thus, it may not be entirely appropriate for AsA (Hwang et al., 2018). Second, CBT skills training sometimes emphasizes independence, assertiveness, and interpersonal confrontations, which might be in conflict with some AsA social norms and values (e.g., interpersonal harmony; Lin, 2002). Third, although AsA tend to exhibit respect towards authorities, they might also be reluctant to express their concerns to therapists, potentially undermining collaboration and therapeutic alliance, and possibly resulting in early termination (Chen \& Davenport, 2005). 


\section{Methods}

\section{Systematic Narrative Review}

A systematic narrative review approach was chosen because it allows for the presentation of both qualitative and quantitative results and is appropriate for small samples of studies with specific groups (Popay et al., 2006). Key elements of narrative synthesis were employed (Popay et al., 2006; develop a theory; synthesize relevant research; explore patterns within and across studies). First, we reviewed literature relevant to a theory about why and for whom interventions work, with a focus on CBT for AsA, and the overlap between AsA cultural values and CBT components. Based on this review, we expected that both culturally-adapted and non-adapted CBT would be effective for AsA youth. To examine this possibility, we then synthesized and explored patterns of findings within and across these studies.

\section{Literature Search}

All procedures were conducted in accordance with PRISMA. The search was conducted from August 2020 to February 2021, with no publication date restrictions. We used four search engines (PsycINFO, Web of Science, PubMed, ProQuest) to identify peer-reviewed academic articles written in English. Our search terms fell under three broad domains: the AsA population, CBT, and youth (see Supplementary Materials 1 for specifics). Terms referencing cognitive, but not behavioral, approaches were included, as we were primarily interested in treatments involving cognitive techniques, given their theorized overlap with AsA values. The first search was conducted to exclude studies with pharmacotherapy. This search yielded 8 studies. We then expanded the search to include studies that involved CBT combined with pharmacotherapy in order to potentially enlarge the study sample. Specifically, two authors independently searched for studies of both CBT and pharmacotherapy (e.g., medication). This search yielded no 
additional studies. Finally, we searched related youth psychotherapy reviews and meta-analyses (e.g., Huey \& Tilley, 2018; Weisz et al., 2019) and followed reference trails to confirm that no eligible studies were missed in our search.

\section{Inclusion and Exclusion Criteria}

Studies were included if they featured: (a) an intervention or prevention condition with at least one cognitive technique, (b) an RCT design, (c) a control condition, (d) youth participants between the ages of 5 to 24 years, (e) a measured outcome specific to the child (rather than only measures focusing on caregiver or family outcomes), (e) only participants who identified as Asian living in the United States at the time of the study, or analyses of treatment effects for AsA youth within a racially diverse sample, and (f) an intervention designed to prevent or treat problems related to one or more psychiatric disorders (American Psychiatric Association, 2013). Studies were excluded if they only reported data from follow-up assessments (see Figure 1).

\section{Risk of Bias}

Using Cochrane's Risk of Bias tool, two authors independently assessed adequate (1) generation of allocation sequence, (2) concealment of condition allocation, (3) concealment from study assessors, and (4) method of addressing incomplete data (mean kappa $=.68$ ), with discrepancies resolved by a third author. According to these criteria, no studies were rated as low-risk, five were rated as having some concerns, and three were rated as high-risk.

\section{Results}

\section{Search Results}

Of the 2,083 potentially relevant articles screened, seven met the criteria, with one article including two studies. Thus, eight studies were included. Given the small number of studies yielded from our extensive search, quantitative meta-analyses could not be reliably conducted. 


\section{Synthesis of Study Characteristics Among Studies}

The eight included RCTs varied widely across: sample size $(N=15-426)$, age of youth participants ( $M=8.4-23.5$ years), and targeted problems (see Table 1). Overall, CBT was effective across these eight treatment studies in reducing targeted problems for AsA youth and exhibited stronger effects compared to the control group. Average effect sizes (ESs) of targeted outcomes within individual studies were small $(n=3)$, medium $(n=1)$, and large $(n=3)$; one study did not report ESs (see Tables S1-S8 for details). Four studies examined samples of exclusively AsA youth, and four compared treatment outcomes across different racial and/or ethnic groups including AsA youth (AsA representation range: $8-100 \%$ of broader sample). Three studies tested a culturally-adapted CBT intervention (details below). Regarding therapy format, one intervention was dyadic and web-based, and all others were in-person, including four group treatments, two individual treatments, and one mixed group and individual intervention. Control groups included waitlist $(n=3)$, no intervention $(n=1)$, and self-help with educational materials (e.g., videos, manuals; $n=4$ ). Treatment duration ranged from 3 to 14 sessions. Among the five studies reporting therapists' ethnicities, three included exclusively AsA therapists and two included mostly White therapists. Finally, all studies measured targeted outcomes with wellestablished standardized measures (Cronbach's $\alpha$ s: acceptable to very good).

\section{Synthesis of Individual Studies}

\section{Specific Phobia}

Two studies conducted by the same research team evaluated CBT for specific phobia in AsA young adults (Huey \& Pan, 2006; Pan et al., 2011). Both compared the effects of (a) standard one-session treatment (OST-S), (b) culturally adapted one-session treatment (OST-CA), and (c) self-help control. OST-S involves gradual, therapist-directed exposure to anxiety 
arousing stimuli, wherein participants mimic therapist modeling of nonphobic responses to phobic stimuli, coupled with emotional identification. OST-CA was a cultural adaptation of OST-S, involving the identification of cognitions, rather than emotions, and six other researchbased adaptations to intervention content and delivery (e.g., authoritative therapeutic relationship). Both studies found that both OST-S and OST-CA were significantly better in reducing phobia symptoms than the self-help control condition at post-treatment and six-month follow-up, as reflected in large and very large ESs, respectively (see Tables S1 and S2). Finally, results suggested that the adapted treatment (OST-CA) was most beneficial for less acculturated participants.

\section{Eating disorders}

Three studies evaluated the potential clinical benefits of a 4- to 6-session dissonancebased prevention program for eating disorders among AsA youth compared to a self-help control group (Rodriguez et al., 2008; Stice et al., 2014 Studies 1 and 2) and a waitlist control (Stice et al., 2014 Study 2). The program was delivered by study staff (Rodriguez et al., 2008; efficacy trial), school personnel (Rodriguez et al., 2008; effectiveness trial), mental health professionals (Stice et al., 2014; Study 1), and peer leaders (Stice et al., 2014; Study 2). The program involved generating cognitive dissonance by challenging the thin ideal (i.e., the belief that the ideal body type is thin) via verbal, written and behavioral exercises (e.g., writing essays, role-plays). Across studies and varying delivery methods (e.g., by study staff vs. peer leaders), this program was effective in reducing eating disorder symptoms (e.g., body dissatisfaction) compared to control groups, with associated ESs ranging from very small (Stice et al., 2014) to small (Rodriguez et al., 2008; see Tables S3-S5). Effects did not vary significantly across racial subgroups, suggesting that the program was as effective for AsA youth as other racial groups. 


\section{Depression}

Marchand et al. (2010) assessed the effectiveness of a 4-session cognitive-behavioral depression prevention program, relative to a waitlist control, among AsA youth with elevated depressive symptoms. The intervention involved CBT techniques (e.g., cognitive restructuring, behavioral activation) aimed at reducing negative and unrealistic cognitions and increasing engagement in pleasant, fulfilling activities. Researchers found a significantly larger decrease in depressive symptoms among the intervention group, compared to the waitlist control group (small ES; Table S6). Effects did not differ significantly across racial subgroups, suggesting that AsA youth benefited from the treatment as much as other youth.

\section{Substance Abuse}

Fang et al. (2010) evaluated the efficacy of a 9-session cognitive-behavioral web-based substance abuse prevention program, relative to a no-treatment control group, with AsA motherdaughter dyads. The intervention included a variety of interactive cognitive (e.g., reframing) and behavioral techniques (e.g., role-plays, positive reinforcement) designed to enhance substance use prevention skill (e.g., refusal skills) mastery. Parental monitoring was also a key component. ESs were not reported but chi-square tests examining group differences (Table S7) indicated that compared to the control group, girls who completed the prevention program exhibited significantly less substance use (excluding cigarette use), as well as better mental health, substance use prevention skills, and mother-daughter relationship quality.

\section{Behavior Problems}

Lau et al. (2011) evaluated a 14-session culturally-adapted parent training program with Chinese immigrant parents and their children exhibiting behavior problems. Several behavioral parent-training techniques were taught, such as praise, tangible rewards, effective commands, 
and ignoring misbehavior. Cognitive restructuring was also included and involved teaching parents to identify thoughts that contribute to maladaptive parenting techniques (e.g., punitive discipline), such as blaming their child for undesirable behavior, and replacing them with thoughts that contribute to more effective behavior management (e.g., non-blaming thoughts). Cultural adaptations included discussing cultural concerns about the skills (e.g., worry that praise will decrease children's motivation to try hard) and tailoring reward systems to emphasize Asian values, such as filial piety. Relative to the waitlist group, families in the intervention group reported significant decreases in child externalizing and internalizing problems and increases in positive parenting behaviors (medium ESs; Table S8). These gains were maintained at the 6month follow-up assessment.

\section{Discussion}

This review provides a synthesis of existing RCTs testing CBT with AsA youth, suggesting that both culturally-adapted and non-adapted CBT may be beneficial for a range of mental health problems among AsA youth. This finding is consistent with literature on the shared attributes of AsA culture and CBT (Sandil, 2006) and studies of CBT with AsA adults (e.g. Ng \& Wong, 2018). Notably, only eight studies met eligibility criteria, and these exhibited a wide range of study and intervention characteristics, underscoring the need for future CBT trials with racially diverse samples, including AsA youth. Likewise, only two almost-identical studies compared adapted and non-adapted interventions, and both found that culturally-adapted interventions evidenced some superiority, paralleling extant literature (Huey \& Tilley, 2018). The small number of studies appears to be due to both the dearth of studies focused exclusively on AsA youth and the often very small, absent, or unreported AsA subsamples, thus precluding subgroup analyses. Overall, this review suggests that CBT may be helpful for AsA youth, 
however, the notable dearth of literature with this population necessitates future research to assess the extent to which our findings are replicated. Accordingly, the remainder of this review outlines the limitations of our study within the context of the larger literature base, and concludes with three critical directions for future research.

\section{Limitations}

Although included studies provide useful information, large replication trials are needed to determine the validity of their findings (Fang \& Schinke, 2013; Pan et al., 2011). Our limited sample size highlights the relative paucity of research designed to test the effectiveness of CBT, a well-established evidence-based psychotherapy (EBP), among AsA and/or racially diverse youth, ultimately pointing to the need for more concerted efforts dedicated to assessing whether interventions designed by and for White and Western populations are effective for minoritized racial and ethnic communities. Given our specific focus on CBT for AsA youth, we intentionally established relatively narrow inclusion/exclusion criteria; future research could consider expanding these criteria (e.g., CBT studies of Asian youth outside of the U.S.) to increase analytic power. Second, included studies varied widely in design (e.g., types of control groups), intervention delivery (e.g., clinician ethnicity, treatment duration, number of sessions, cultural adaptation), format (e.g., web-based, in-person), and samples (demographic characteristics; target problems). The heterogeneity in intervention characteristics likely contributed to the observed treatment differences. Indeed, a prior meta-analysis on CBT for Asians demonstrated a wide range in effect sizes and found that intervention characteristics moderated CBT efficacy (Ng \& Wong, 2018). This heterogeneity in intervention characteristics, coupled with the small number of studies overall, limits the generality of conclusions that might be drawn (e.g., about the relative efficacy of treatments for one disorder vs. another among AsA youth). Third, 
although this review focuses broadly on AsA youth, it is critical to emphasize that there are numerous ethnicities within this population who exhibit an array of diverse values (Budiman et al., 2019). Additionally, cultural values differ across AsA generations, with Asian values being more actively endorsed by first-generation immigrants compared with later generations (e.g., their children and grandchildren; Ahn et al., 2008). Given the limited number of studies focusing on AsA youth as a whole, it is unsurprising that there is also limited evidence on potentially differential treatment effectiveness across AsA ethnicities (Huey \& Tilley, 2018) and generations. In fact, only two studies in our review reported ethnicities among AsA participants and only two reported the age of immigration to the U.S. In other words, we cannot examine nor determine whether treatment effects differ across ethnic AsA subgroups given the limited research to date.

Finally, while most studies involved EBPs, some did not employ interventions with established EBP protocols that might have provided the most potent treatment (see Table S9 for details). For example, Huey and Pan (2006) tested exposure, the gold-standard method for treating specific phobia, but they only did so in one session, despite research suggesting that multiple-session exposure is maximally effective (Wolitzky-Taylor et al.,2008). Although research on the benefits of single session treatment is growing (Schleider \& Weisz, 2017) and the definition of EBP continues to evolve (Cook et al., 2017), future research may benefit from testing more well-established EBPs with AsA youth.

\section{Future Directions}

Ultimately, our review underscores the critical importance of future research, and we highlight three potential domains of focus. 


\section{Enhance Cultural Responsiveness of Research Practices}

To substantially advance our understanding of EBPs for AsA youth, we must reconsider dominant research practices in intervention science, which were designed by and for White and Western populations (Benish et al., 2011). For instance, prevailing methods of participant recruitment in treatment trials are typically passive and do not involve direct contact with the target population (Liu et al., 2018). Passive recruitment may not be effective for minoritized communities, including AsA, given barriers to research participation that disproportionately affect these groups, such as warranted mistrust of academic and healthcare institutions, linguistic mismatch, and time constraints (Katigbak et al., 2016; David et al., 2010). Instead, experts propose leveraging community-research partnerships (e.g., Collins et al., 2018; Lau et al., 2010) that actively consider the broader, converging socio-political factors impacting research engagement in minoritized communities (e.g., Matching Model of Recruitment; Javier et al., 2019). Once participants are successfully recruited, research practices (e.g., consent procedures, participant payment rates and transactions, assessment and data collection procedures) should be carefully audited and modified in collaboration with community members. For example, culturally responsive researchers should include write-in options for demographic questions (e.g., ethnicity, gender), ask community members about the appropriateness and resonance of included measures, provide appropriate payment (e.g., cash vs. check) that justifies participation over other financially beneficial opportunities, and use flexible scheduling procedures to accommodate a range of employment, transportation, and child care needs (Collins et al., 2018). Finally, researchers should consider cultural variations in symptom expression (e.g., somatic complaints in AsA), and the limitations of using primarily Western diagnostic categories (e.g., in the DSM-5) in research (e.g., as an inclusion criterion; Zhou et al., 2009). 


\section{Enhance Cultural Responsiveness of Evidence-Based Psychotherapies and Therapists}

Despite some evidence for stronger effectiveness of adapted vs. non-adapted treatments for AsA (i.e., in this review and others; e.g., Huey \& Tilley, 2018), the dearth of relevant studies precludes reliable conclusions about their relative utility. Indeed, the development and testing of culturally-adapted treatments involves considerable time and resources, which markedly hinders the expediency of their dissemination (Lund, 2003; Grandpierre et al., 2018). Thus, scholars have recently begun highlighting the utility of more generalizable culturally responsive practices to address disparities in the accessibility and effectiveness of EBPs for AsA and other minoritized youth (Belone et al., 2016). Culturally responsive practice involves training providers in the unique values and needs of particular groups (e.g., specific ethnic groups), as well as how to leverage this knowledge to modify existing practices (Kim-Goh et al., 2015; Zigarelli et al., 2016). To identify optimal culturally responsive practices, it is critical to understand the values and needs of community stakeholders (e.g., service providers, community leaders) through community-engaged research (i.e., research created and conducted by close community researchers to seek to help or understand; Wallerstein \& Duran, 2006). This process is illustrated by Wang, Shao, et al. (2019), who invited community leaders, AsA youth, and parents to identify their mental health needs and challenges through interviews and personal essays. These qualitative data revealed key targets for improving the accessibility (e.g., cultural stigma toward support seeking, communication barriers) and effectiveness (e.g., recognizing AsA family strengths, promoting parent empathy) of EBPs for AsA youth and families. This study exemplifies the utility of employing community-engaged research methods to optimize accessibility to, and effectiveness of, EBPs for AsA youth. 


\section{Reducing Barriers to Mental Health Service Access and Utilization}

Although understanding the effectiveness of EBPs for AsA youth is important, AsA and other minoritized communities face a variety of barriers to accessing and engaging in any mental health care (Yang et al., 2019). Of note, there is great heterogeneity in treatment access barriers within larger racial groups. For instance, Filipino, Japanese, and Indian Americans have relatively higher English proficiency than other AsA groups (e.g., Chinese, Vietnamese, Korean), thus making mental health care access in the $\underline{\text { U.S. }}$ especially difficult for some AsA groups (Kang et al., 2010; Sorkin et al., 2011). Accordingly, research employing communityengaged methods to understand and support a variety of AsA communities in overcoming unique and shared barriers to mental health service use is essential (Lee et al., 2019). For example, through focus groups with AsA young adults representing eight ethnic groups, Lee et al. (2009) found that AsA youth hesitate to access mental health treatment because they do not want to alarm their parents, and due to their parents' lack of mental health knowledge. Thus, participants suggested that providing psychoeducation to parents about how to effectively discuss mental health with their children would empower youth and families to seek mental health services. This example illustrates the utility of community voices in both the adaptation of services (e.g., adding parent psychoeducation) and the successful implementation of services (i.e., overcoming barriers). Indeed, scholars have recently begun developing and testing efficient communityengaged intervention tailoring methods that target both efficacy and implementation (e.g., human-centered design; Lyon \& Bruns, 2019).

In sum, promising community-engaged research methods have been developed and recently deployed to address mental health service needs for populations that have been historically underrepresented in clinical research and poorly served in real-world settings. 
Moving forward, increased use of these methods and rapid, large-scale deployment of resulting findings will be essential in reducing mental health inequities for minoritized communities, including AsA youth. To be successful, such efforts first require researchers to carefully consider (1) their application of dominant approaches to intervention science and psychological research more broadly, which center Whiteness and Western values, (2) how these approaches may be misaligned with the goal of advancing equity in the development, assessment, and dissemination of youth psychotherapy, and (3) how the integration of community-engaged research methods into the field of intervention science can better advance this goal, within and beyond the context of EBPs for AsA youth and families. 


\section{References}

Abe-Kim, J., Takeuchi, D. T., Hong, S., Zane, N., Sue, S., Spencer, M. S., Appel, H., Nicdao, E., \& Alegría, M. (2007). Use of mental health-related services among immigrant and USborn Asian Americans: Results from the national Latino and Asian American study. American Journal of Public Health, 97(1), 91-98.

https://doi.org/10.2105/AJPH.2006.098541

Ahn, A. J., Kim, B. S. K., \& Park, Y. S. (2008). Asian cultural values gap, cognitive flexibility, coping strategies, and parent-child conflicts among Korean Americans. Cultural Diversity and Ethnic Minority Psychology, 14(4), 353-363. https://doi.org/10.1037/10999809.14.4.353

American Psychiatric Association. (2013). Diagnostic and statistical manual of mental disorders (5th ed.). https://doi.org/10.1176/appi.books.9780890425596

Atkinson, D. R., Maruyama, M., \& Matsui, S. (1978). Effects of counselor race and counseling approach on Asian Americans' perceptions of counselor credibility and utility. Journal of Counseling Psychology, 25, 76-83.

Beck, A. T., Rush, A. J., Shaw, B. F., \& Emery, G. (1979). Cognitive therapy of depression. Guilford Press.

Belone, L., Tosa, J., Shendo, K., Toya, A., Straits, K., Tafoya, G., Rae, R., Noyes, E., Bird, D., \& Wallerstein N. (2016). Community based participatory research (CBPR) principles and strategies for co-creating culturally-centered interventions with native communities: A partnership between the University of New Mexico and the Pueblo of Jemez with implications for other ethnocultural communities. In: N. Zane, G. Bernal, F. Leong (Eds.), Evidence-based psychological practice with ethnic minorities: Culturally 
informed research and clinical strategies (pp. 199-220). American Psychological Association.

Benish, S. G., Quintana, S., \& Wampold, B. E. (2011). Culturally adapted psychotherapy and the legitimacy of myth: A direct-comparison meta-analysis. Journal of Counseling Psychology, 58(3), 279-289. https://doi.org/10.1037/a0023626

Budiman, A., Cilluffo, A., \& Ruiz, N. G. (2019, May 22). Key facts about Asian origin groups in the U.S. Pew Research Center. https://www.pewresearch.org/fact-tank/2019/05/22/keyfacts-about-asian-origin-groups-in-the-u-s/

Chen, S. W. H., \& Davenport, D. S. (2005). Cognitive-behavioral therapy with Chinese American clients: Cautions and modifications. Psychotherapy: Theory, Research, Practice, Training, 42(1), 101-110. https://doi.org/10.1037/0033-3204.42.1.101

Cheng, Z. H., Perko, V. L., Fuller-Marashi, L., Gau, J. M., \& Stice, E. (2019). Ethnic differences in eating disorder prevalence, risk factors, and predictive effects of risk factors among young women. Eating Behaviors, 32, 23-30. https://doi.org/10.1016/j.eatbeh.2018.11.004

Chiang, S., Chin, C. A., Meyer, E. W., Sust, S., \& Chu, J. (2021). Asian American adolescent help-seeking pathways for psychological distress. Asian American Journal of Psychology. Advance online publication. https://doi.org/10.1037/aap0000241

Choi, Y., \& Lahey, B. B. (2006). Testing the model minority stereotype: Youth behaviors across racial and ethnic groups. Social Service Review, 80(3), 419-452. https://doi.org/10.1086/505288

Chu, B. C., \& Harrison, T. L. (2007). Disorder-specific effects of CBT for anxious and depressed youth: A meta-analysis of candidate mediators of change. Clinical Child and Family Psychology Review, 10(4), 352-372. https://doi.org/10.1007/s10567-007-0028-2 
Collins, S. E., Clifasefi, S. L., Stanton, J., Board, T. L. A., Straits, K. J. E., Gil-Kashiwabara, E., Rodriguez Espinosa, P., Nicasio, A. V, Andrasik, M. P., Hawes, S. M., Miller, K. A., Nelson, L. A., Orfaly, V. E., Duran, B. M., \& Wallerstein, N. (2018). Community-based participatory research (CBPR): Towards equitable involvement of community in psychology research. The American Psychologist, 73(7), 884-898. https://doi.org/10.1037/amp0000167

Cook, S. C., Schwartz, A. C., \& Kaslow, N. J. (2017). Evidence-based psychotherapy: Advantages and challenges. Neurotherapeutics, 14(3), 537-545. https://doi.org/10.1007/s13311-017-0549-4

David, E. J. R. (2010). Cultural mistrust and mental health help-seeking attitudes among Filipino Americans. Asian American Journal of Psychology, 1(1), 57-66. https://doi.org/10.1037/a0018814

Exum, H. A., \& Lau, E. Y. (1988). Counseling style preference of Chinese college students. Journal of Multicultural Counseling and Development, 16(2), 84-92. https://doi.org/10.1002/j.2161-1912.1988.tb00644.x

Fang, L., \& Schinke, S. P. (2013). Two-year outcomes of a randomized, family-based substance use prevention trial for Asian American adolescent girls. Psychology of Addictive Behaviors, 27(3), 788-798. https://doi.org/10.1037/a0030925

Fang, L., Schinke, S. P., \& Cole, K. C. A. (2010). Preventing substance use among early AsianAmerican adolescent girls: Initial evaluation of a web-based, mother-daughter program. Journal of Adolescent Health, 47(5), 529-532. https://doi.org/10.1016/j.jadohealth.2010.03.011 
Gee, G. C., Spencer, M. S., Chen, J., \& Takeuchi, D. (2007). A Nationwide study of discrimination and chronic health conditions among Asian Americans. American Journal of Public Health, 97(7), 1275-1282. https://doi.org/10.2105/AJPH.2006.091827

Guo, S., Kataoka, S. H., Bear, L., \& Lau, A. S. (2014). Differences in school-based referrals for mental health care: Understanding racial/ethnic disparities between Asian American and Latino youth. School Mental Health, 6(1), 27-39. https://doi.org/10.1007/s12310-0139108-2

Grandpierre, V., Milloy, V., Sikora, L., Fitzpatrick, E., Thomas, R., \& Potter, B. (2018). Barriers and facilitators to cultural competence in rehabilitation services: a scoping review. $B M C$ health services research, 18(1), 1-14. https://doi.org/10.1186/s12913-017-2811-1

Haine-Schlagel, R., \& Walsh, N. E. (2015). A review of parent participation engagement in child and family mental health treatment. Clinical Child and Family Psychology Review, 18(2), 133-150. https://doi.org/10.1007/s10567-015-0182-x

Hall, G. C. N., Kim-Mozeleski, J. E., Zane, N. W., Sato, H., Huang, E. R., Tuan, M., \& Ibaraki, A. Y. (2019). Cultural adaptations of psychotherapy: Therapists' applications of conceptual models with Asians and Asian Americans. Asian American Journal of Psychology, 10(1), 68-78. https://doi.org/10.1037/aap0000122

Heras, P. (2007). Psychotherapy with Filipinas. Women \& Therapy, 30(3-4), 63-73. https://doi.org/10.1300/J015v30n03_06

Hsieh, A. L., \& Bean, R. A. (2014). Understanding familial/cultural factors in adolescent depression: A culturally-competent treatment for working with Chinese American families. The American Journal of Family Therapy, 42(5), 398-412. https://doi.org/10.1080/01926187.2014.884414 
Hua, J. M., \& Costigan, C. L. (2012). The familial context of adolescent language brokering within immigrant Chinese families in Canada. Journal of Youth and Adolescence, 41(7), 894-906. https://doi.org/10.1007/s10964-011-9682-2

Huey, S. Jr., \& Pan, D. (2006). Culture-responsive one-session treatment for phobic Asian Americans: A pilot study. Psychotherapy: Theory, Research, Practice, Training, 43(4), 549-554. https://doi.org/10.1037/0033-3204.43.4.549

Huey, S. J., \& Tilley, J. L. (2018). Effects of mental health interventions with Asian Americans: A review and meta-analysis. Journal of Consulting and Clinical Psychology, 86(11), 915-930. https://doi.org/10.1037/ccp0000346

Hwang, W. C., Ho, L. C., Chan, C. P., \& Hong, K. K. (2018). Cognitive behavioral models, measures, and treatments for depressive disorders in Asian Americans. In E. C. Chang, C. A. Downey, J. K. Hirsch, \& E. A. Yu (Eds.), Treating depression, anxiety, and stress in ethnic and racial groups: Cognitive behavioral approaches (Cultural, racial, and ethnic psychology book series, pp. 23-47). American Psychological Association.

Hwang, W. C., Wood, J. J., Lin, K. M., \& Cheung, F. (2006). Cognitive-behavioral therapy with Chinese Americans: Research, theory, and clinical practice. Cognitive and Behavioral Practice, 13(4), 293-303. https://doi.org/10.1016/j.cbpra.2006.04.010

Hynes, K. C. (2019). Cultural values matter: The therapeutic alliance with East Asian Americans. Contemporary Family Therapy, 41(4), 392-400. https://doi.org/10.1007/s10591-019-09506-9

Iwamasa, G. Y., Hsia, C., \& Hinton, D. (2019). Cognitive behavior therapy with Asian Americans. In G. Y. Iwamasa \& P. A. Hays (Eds.), Culturally responsive cognitive 
behavior therapy: Practice and supervision (2nd ed., pp. 129-159). American Psychological Association.

Jaiswal, J., \& Halkitis, P. N. (2019). Towards a more inclusive and dynamic understanding of medical mistrust informed by science. Behavioral Medicine, 45(2), 79-85. https://doi.org/10.1080/08964289.2019.1619511

Javier, J. R., Galura, K., Aliganga, F. A. P., Supan, J., \& Palinkas, L. A. (2018). Voices of the Filipino community describing the importance of family in understanding adolescent behavioral health needs. Family \& Community Health: The Journal of Health Promotion \& Maintenance, 41(1), 64-71. https://doi.org/10.1097/FCH.0000000000000173

Javier, J. R., Reyes, A., Coffey, D. M., Schrager, S. M., Samson, A., Palinkas, L., Kipke, M. D., \& Miranda, J. (2019). Recruiting Filipino immigrants in a randomized controlled trial promoting enrollment in an evidence-based parenting intervention. Journal of Immigrant and Minority Health, 21(2), 324-331. https://doi.org/10.1007/s10903-018-0755-0

Kang, S. Y., Howard, D., Kim, J., Payne, J. S., Wilton, L., Kim, W., \& Maramba, D. (2010). English language proficiency and lifetime mental health service utilization in a national representative sample of Asian Americans in the USA. Journal of Public Health, 32(3), 431-439. https://doi.org/10.1093/pubmed/fdq010

Katigbak, C., Foley, M., Robert, L., \& Hutchinson, M. (2016). Experiences and lessons learned in using community-based participatory research to recruit Asian American immigrant research participants. Journal of Nursing Scholarship, 48. https://doi.org/10.1111/jnu.12194 
Khanna, M. S., \& Kendall, P. C. (2009). Exploring the role of parent training in the treatment of childhood anxiety. Journal of Consulting and Clinical Psychology, 77(5), 981-986. https://doi.org/10.1037/a0016920

Kim, B. S. K., Yang, P. H., Atkinson, D. R., Wolfe, M. M., \& Hong, S. (2001). Cultural value similarities and differences among Asian American ethnic groups. Cultural Diversity and Ethnic Minority Psychology, 7(4), 343-361. https://doi.org/10.1037/1099-9809.7.4.343

Kim-Goh, M., Choi, H., \& Yoon, M. S. (2015). Culturally responsive counseling for Asian Americans: Clinician perspectives. International Journal for the Advancement of Counselling, 37(1), 63-76. https://doi.org/10.1007/s10447-014-9226-z

Lau, A. S., Chang, D. F., \& Okazaki, S. (2010). Methodological challenges in treatment outcome research with ethnic minorities. Cultural Diversity \& Ethnic Minority Psychology, 16(4), 573-580. https://doi.org/10.1037/a0021371

Lau, A. S., Fung, J. J., Ho, L. Y., Liu, L. L., \& Gudiño, O. G. (2011). Parent training with highrisk immigrant Chinese families: A pilot group randomized trial yielding practice-based evidence. Behavior Therapy, 42(3), 413-426. https://doi.org/10.1016/j.beth.2010.11.001

Le Meyer, O., Zane, N., Cho, Y. I., \& Takeuchi, D. T. (2009). Use of specialty mental health services by Asian Americans with psychiatric disorders. Journal of Consulting and Clinical Psychology, 77(5), 1000-1005. https://doi.org/10.1037/a0017065

Lee, D. L., \& Ahn, S. (2011). Racial discrimination and Asian Mental Health: A meta-analysis. The Counseling Psychologist, 39(3), 463-489. https://doi.org/10.1177/0011000010381791

Lee, S., Juon, H. S., Martinez, G., Hsu, C. E., Robinson, E. S., Bawa, J., \& Ma, G. X. (2009). Model minority at risk: Expressed needs of mental health by Asian American young 
adults. Journal of Community Health, 34(2), 144-152. https://doi.org/10.1007/s10900008-9137-1

Leong, F. T. (1986). Counseling and psychotherapy with Asian-Americans: Review of the literature. Journal of Counseling Psychology, 33(2), 196-206. https://doi.org/10.1037/0022-0167.33.2.196

Leong, F. T. L., \& Lau, A. S. L. (2001). Barriers to providing effective mental health services to Asian Americans. Mental Health Services Research, 3(4). https://doi.org/ 10.1023/a:1013177014788

Li, L. C., \& Kim, B. S. K. (2004). Effects of counseling style and client adherence to Asian cultural values on counseling process with Asian American college students. Journal of Counseling Psychology, 51(2), 158-167. https://doi.org/10.1037/0022-0167.51.2.158

Lin, Y. -N. (2002). The application of cognitive-behavioral therapy to counseling Chinese. American Journal of Psychotherapy, 56(1), 46-58. https://doi.org/10.1176/appi.psychotherapy.2002.56.1.46

Ling, A., Okazaki, S., Tu, M. -C., \& Kim, J. J. (2014). Challenges in meeting the mental health needs of urban Asian American adolescents: Service providers' perspectives. Race and Social Problems, 6(1), 25-37. https://doi.org/10.1007/s12552-014-9117-2

Liu, Y., Pencheon, E., Hunter, R. M., Moncrieff, J., \& Freemantle, N. (2018). Recruitment and retention strategies in mental health trials - A systematic review. PloS One, 13(8), e0203127. https://doi.org/10.1371/journal.pone.0203127

López, G., Ruiz, N. G., \& Patten, E. (2017, September 8). Key facts about Asian Americans, a diverse and growing population. Pew Research Center. https://www.pewresearch.org/fact-tank/2017/09/08/key-facts-about-asian-americans/ 
Lund, C. (2003). Planning and Budgeting to Deliver Services for Mental Health (Vol. 3). World Health Organization.

Lyon, A. R., \& Bruns, E. J. (2019). User-centered redesign of evidence-based psychosocial interventions to enhance implementation-hospitable soil or better seeds? JAMA Psychiatry, 76(1), 3. https://doi.org/10.1001/jamapsychiatry.2018.3060

Marchand, E., Ng, J., Rohde, P., \& Stice, E. (2010). Effects of an indicated cognitive-behavioral depression prevention program are similar for Asian American, Latino, and European American adolescents. Behaviour Research and Therapy, 48(8), 821-825. https://doi.org/10.1016/j.brat.2010.05.005

McCarty, C. A., Weisz, J. R., Wanitromanee, K., Eastman, K. L., Suwanlert, S., Chaiyasit, W., \& Band, E. B. (1999). Culture, coping, and context: Primary and secondary control among Thai and American youth. Journal of Child Psychology and Psychiatry, 40(5), 809-818. https://doi.org/10.1111/1469-7610.00496

McMurtry, C. L., Findling, M. G., Casey, L. S., Blendon, R. J., Benson, J. M., Sayde, J. M., \& Miller, C. (2019). Discrimination in the United States: Experiences of Asian Americans. Health Services Research, 54(S2), 1419-1430. https://doi.org/10.1111/1475-6773.13225

Misra, S., Jackson, V. W., Chong, J., Choe, K., Tay, C., Wong, J., \& Yang, L. H. (2021). Systematic review of cultural aspects of stigma and mental illness among racial and ethnic minority groups in the United States: Implications for interventions. American Journal of Community Psychology. https://doi.org/10.1002/ajcp.12516

Ng, T. K., \& Wong, D. F. K. (2018). The efficacy of cognitive behavioral therapy for Chinese people: A meta-analysis. Australian \& New Zealand Journal of Psychiatry, 52(7), 620637. https://doi.org/10.1177/0004867417741555 
O’Donohue, W., \& Benuto, L. (2010). The many problems of cultural sensitivity. The Scientific Review of Mental Health Practice, 7(2), 34-37.

Pan, D., Huey, S. J., \& Hernandez, D. (2011). Culturally adapted versus standard exposure treatment for phobic Asian Americans: Treatment efficacy, moderators, and predictors. Cultural Diversity and Ethnic Minority Psychology, 17(1), 11-22. https://doi.org/10.1037/a0022534

Popay, J., Roberts, H., Sowden, A., Petticrew, M., Arai, L., Britten, N., Rodgers, M., Roen, K., \& Duffy, S. (2006). Guidance on the conduct of narrative synthesis in systematic reviews. ESRC Methods Programme.

Qin, D. B., Way, N., \& Mukherjee, P. (2008). The other side of the model minority story: The familial and peer challenges faced by Chinese American adolescents. Youth \& Society, 39(4), 480-506. https://doi.org/10.1177/0044118X08314233

Rodriguez, R., Marchand, E., Ng, J., \& Stice, E. (2008). Effects of a cognitive dissonance-based eating disorder prevention program are similar for Asian American, Hispanic, and White participants. International Journal of Eating Disorders, 41(7), 618-625. https://doi.org/10.1002/eat.20532

Rothbaum, F., Weisz, J. R., \& Snyder, S. S. (1982). Changing the world and changing the self: A two-process model of perceived control. Journal of Personality and Social Psychology, 42(1), 5-37. https://doi.org/10.1037/0022-3514.42.1.5

Sandil, R. (2006). Cognitive behavioral therapy for adolescent depression: Implications for Asian immigrants in the United States of America. Journal of Child \& Adolescent Mental Health, 18(1), 27-32. https://doi.org/10.2989/17280580609486615 
Schleider, J. L., \& Weisz, J. R. (2017). Little treatments, promising effects? Meta-Analysis of single-session interventions for youth psychiatric problems. Journal of the American Academy of Child \& Adolescent Psychiatry, 56(2), 107-115.

https://doi.org/10.1016/j.jaac.2016.11.007

Sentell, T., Shumway, M., \& Snowden, L. (2007). Access to mental health treatment by English language proficiency and race/ethnicity. Journal of General Internal Medicine, 22(Suppl 2), 289-293. https://doi.org/10.1007/s11606-007-0345-7

Stice, E., Marti, C. N., \& Cheng, Z. H. (2014). Effectiveness of a dissonance-based eating disorder prevention program for ethnic groups in two randomized controlled trials. Behaviour Research and Therapy, 55, 54-64. https://doi.org/10.1016/j.brat.2014.02.002

Song, S. J., Ziegler, R., Arsenault, L., Fried, L. E., \& Hacker, K. (2011). Asian student depression in American high schools: Differences in risk factors. Journal of School Nursing, 27(6), 455-462. https://doi.org/10.1177/1059840511418670

Sorkin, D. H., Nguyen, H., \& Ngo-Metzger, Q. (2011). Assessing the mental health needs and barriers to care among a diverse sample of Asian American older adults. Journal of general internal medicine, 26(6), 595-602. https://doi.org/10.1007/s11606-010-1612-6

Sotto-Santiago, S. (2019). Time to reconsider the word minority in academic medicine. Journal of Best Practices in Health Professions Diversity, 12(1), 72-78.

Sue, D. W., Capodilupo, C. M., Torino, G. C., Bucceri, J. M., Holder, A. M. B., Nadal, K. L., \& Esquilin, M. (2007). Racial microaggressions in everyday life: Implications for clinical practice. American Psychologist, 62(4), 271-286. https://doi.org/10.1037/0003066X.62.4.271 
Tarrier, N., Taylor, K., \& Gooding, P. (2008). Cognitive-behavioral interventions to reduce suicide behavior: A systematic review and meta-analysis. Behavior Modification, 32(1), 77-108. https://doi.org/10.1177/0145445507304728

Uba, L., (2003). Asian-Americans: Personality patterns, identity, and mental health. Guilford Press.

Wallerstein, N. B., \& Duran, B. (2006). Using community-based participatory research to address health disparities. Health promotion practice, 7(3), 312-323. https://doi.org/10.1177/1524839906289376

Wang, C., \& Atwal, K. (2015). School climate, discrimination, and depressive symptoms among Asian American adolescents. Contemporary School Psychology, 19(3), 205-217. https://doi.org/10.1007/s40688-014-0040-8

Wang, C., Do, K. A., Frese, K., \& Zheng, L. (2019). Asian immigrant parents' perception of barriers preventing adolescents from seeking school-based mental health services. School Mental Health, 11(2), 364-377. https://doi.org/10.1007/s12310-018-9285-0

Wang, C., Shao, X., Do, K. A., Lu, H. K., O’Neal, C. R., \& Zhang, Y. (2019). Using participatory culture-specific consultation with Asian American communities: Identifying challenges and solutions for Asian American immigrant families. Journal of Educational and Psychological Consultation, 31(1), 17-38. https://doi.org/10.1080/10474412.2019.1614453

Weisz, J. R., Kuppens, S., Ng, M. Y., Eckshtain, D., Ugueto, A. M., Vaughn-Coaxum, R., Jensen-Doss, A., Hawley, K. M., Krumholz Marchette, L. S., Chu, B. C., Weersing, V. R., \& Fordwood, S. R. (2017). What five decades of research tells us about the effects of 
youth psychological therapy: A multilevel meta-analysis and implications for science and practice. American Psychologist, 72(2), 79-117. https://doi.org/10.1037/a0040360

Weisz, J. R., Kuppens, S., Ng, M. Y., Vaughn-Coaxum, R. A., Ugueto, A. M., Eckshtain, D., \& Corteselli, K. A. (2019). Are psychotherapies for young people growing stronger? Tracking trends over time for youth anxiety, depression, ADHD, and conduct problems. Perspectives on Psychological Science, 14 (2), 216-237. https://doi.org/10.1177/1745691618805436

Wolitzky-Taylor, K. B., Horowitz, J. D., Powers, M. B., \& Telch, M. J. (2008). Psychological approaches in the treatment of specific phobias: A meta-analysis. Clinical Psychology Review, 28(6), 1021-1037. https://doi.org/10.1016/j.cpr.2008.02.007

Wu, C., Qian, Y., \& Wilkes, R. (2020). Anti-Asian discrimination and the Asian-White mental health gap during COVID-19. Ethnic and Racial Studies, 44(5), 819-835. https://doi.org/10.1080/01419870.2020.1851739

Yang, K. G., Rodgers, C. R. R., Lee, E., \& Lê Cook, B. (2019). Disparities in mental health care utilization and perceived need among Asian Americans: 2012-2016. Psychiatric Services, 71(1), 21-27. https://doi.org/10.1176/appi.ps.201900126

Young, C. B. (2010). Depression in Asian-American and Caucasian undergraduate students. Journal of Affective Disorders, 4. http://doi.org/10.1016/j.jad.2010.02.124

Zhou, Z., Siu, C. R., \& Xin, T. (2009). Promoting cultural competence in counseling Asian American children and adolescents. Psychology in the Schools, 46(3), 290-298. https://doi.org/10.1002/pits.20375

Zigarelli, J. C., Jones, J. M., Palomino, C. I., \& Kawamura, R. (2016). Culturally responsive cognitive behavioral therapy: Making the case for integrating cultural factors in evidence- 
based treatment. Clinical Case Studies, 15(6), 427-442.

https://doi.org/10.1177/1534650116664984 
Table 1

Study Characteristics

\begin{tabular}{|c|c|c|c|c|c|c|c|c|c|}
\hline \multirow[b]{2}{*}{$\begin{array}{l}\text { Authors, } \\
\text { Date }\end{array}$} & \multicolumn{4}{|c|}{ Sample characteristics } & \multirow[b]{2}{*}{$\begin{array}{l}\% \text { Clinician } \\
\text { Race / } \\
\text { Ethnicity }(N)\end{array}$} & \multirow[b]{2}{*}{$\begin{array}{l}\text { Control } \\
\text { Group }\end{array}$} & \multicolumn{2}{|c|}{ Treatment characteristics } & \multirow{2}{*}{$\begin{array}{c}\text { ES of } \\
\text { Targeted } \\
\text { Problem } \\
\text { Measures }\end{array}$} \\
\hline & $\begin{array}{l}\text { Size, } \\
\text { Description } \\
\left(M_{\text {Age }}, S D\right)\end{array}$ & $\begin{array}{l}\text { \% Race } \\
\text { (Ethnicities) }\end{array}$ & Immigration & $\begin{array}{l}\text { Cultural } \\
\text { Identity }\end{array}$ & & & $\begin{array}{l}\text { Cultural } \\
\text { Adaptation }\end{array}$ & $\begin{array}{l}\text { Target } \\
\text { Problem }\end{array}$ & \\
\hline $\begin{array}{l}\text { Huey \& } \\
\text { Pan, } 2006\end{array}$ & $\begin{array}{l}15 \text { AsA } \\
\text { college } \\
\text { students } \\
(23.5)\end{array}$ & $\begin{array}{l}100 \text { AsA } \\
\text { (60 Chinese or } \\
\text { Taiwanese, } 13 \\
\text { Japanese, } 13 \\
\text { multiethnic, } 7 \\
\text { Korean, } 7 \\
\text { Vietnamese) }\end{array}$ & $\begin{array}{l}\text { Age at } \\
\text { immigration } \\
1-18 \text { years }\end{array}$ & $\begin{array}{l}47 \% \\
\text { bicultural, } \\
27 \% \text { mostly } \\
\text { Asian, } 13 \% \\
\text { mostly and } \\
13 \% \text { very } \\
\text { Westernized }\end{array}$ & 100 AsA (1) & $\begin{array}{l}\text { Mastery of } \\
\text { Your } \\
\text { Specific } \\
\text { Phobia }\end{array}$ & Yes & $\begin{array}{l}\text { Specific } \\
\text { phobia }\end{array}$ & $\begin{array}{l}\text { Large - } \\
\text { very } \\
\text { large }\end{array}$ \\
\hline $\begin{array}{l}\text { Pan et al., } \\
2011 \\
\text { [follow } \\
\text { up to Huey } \\
\& \text { Pan, } \\
\text { 2006] }\end{array}$ & $\begin{array}{l}30 \text { AsA } \\
\text { college } \\
\text { students } \\
(22.1)\end{array}$ & 100 AsA & & $\begin{array}{l}\text { Medium } \\
\text { acculturation }^{\mathrm{a}}\end{array}$ & 100 AsA (1) & $\begin{array}{l}\text { Mastery of } \\
\text { Your } \\
\text { Specific } \\
\text { Phobia }\end{array}$ & Yes & $\begin{array}{l}\text { Specific } \\
\text { phobia }\end{array}$ & Large \\
\hline $\begin{array}{l}\text { Stice et } \\
\text { al., } 2014 \\
\text { (Study 1) }\end{array}$ & $\begin{array}{l}426 \text { female } \\
\text { college } \\
\text { students } \\
(21.6,5.6)\end{array}$ & $\begin{array}{l}\text { 17AsA', } 6 \\
\text { AfA', } 65 \\
\text { White', } 12 \\
\text { Hispanic' }\end{array}$ & & & $\begin{array}{l}\text { 97 White } \\
(26)\end{array}$ & $\begin{array}{l}\text { Healthy } \\
\text { body } \\
\text { image } \\
\text { brochure } \\
\text { or } \\
\text { video }\end{array}$ & No & $\begin{array}{l}\text { Eating } \\
\text { disorders }\end{array}$ & $\begin{array}{l}\text { Very } \\
\text { small }\end{array}$ \\
\hline $\begin{array}{l}\text { Stice et al., } \\
2014 \\
\text { (Study 2) }\end{array}$ & $\begin{array}{l}189 \text { female } \\
\text { college } \\
\text { students } \\
(20.9,3.8)\end{array}$ & $\begin{array}{l}30 \text { AsA', } \\
\text { 7AfA', } \\
\text { 42White', } 21 \\
\text { Hispanic' }\end{array}$ & & & $\begin{array}{l}8 \text { AfA; } 38 \\
\text { White; } 29 \\
\text { AsA; } 25 \\
\text { Hispanic } \\
(24)\end{array}$ & Waitlist & No & $\begin{array}{l}\text { Eating } \\
\text { disorders }\end{array}$ & $\begin{array}{l}\text { Very } \\
\text { small }\end{array}$ \\
\hline
\end{tabular}




\begin{tabular}{|c|c|c|c|c|c|c|c|c|}
\hline $\begin{array}{l}\text { Rodriguez } \\
\text { et al., } 2008\end{array}$ & $\begin{array}{l}405 \text { female } \\
10 \text { th grade } \\
\text { students } \\
\left(16.8^{\prime},\right. \\
\left.1.45^{\prime}\right)\end{array}$ & $\begin{array}{l}77 \text { White', } 15 \\
\text { Hispanic', } 8 \\
\text { AsA' }\end{array}$ & & & $\begin{array}{l}\text { Self-help } \\
\text { or } \\
\text { assessment } \\
\text {-only }\end{array}$ & No & $\begin{array}{l}\text { Eating } \\
\text { disorder }\end{array}$ & Small \\
\hline $\begin{array}{l}\text { Marchand } \\
\text { et al., } 2010 \\
(\text { Study } 1)^{\mathrm{b}}\end{array}$ & $\begin{array}{l}167 \text { high } \\
\text { school and } \\
\text { college } \\
\text { students } \\
(18.6,1.8) \\
\end{array}$ & \multicolumn{3}{|l|}{$\begin{array}{l}\text { 22AsA/Pacific } \\
\text { Islanders, } 19 \\
\text { Latinos, } \\
\text { 59White }\end{array}$} & Waitlist & No & Depression & Small \\
\hline $\begin{array}{l}\text { Fang et al., } \\
2010\end{array}$ & $\begin{array}{l}108 \text { AsA } \\
\text { girls }(12.6) \\
\text { and mothers } \\
\left(40.2^{\prime}\right)\end{array}$ & \multicolumn{3}{|l|}{$100 \mathrm{AsA}$} & $\begin{array}{l}\text { No } \\
\text { treatment }\end{array}$ & No & \multicolumn{2}{|l|}{$\begin{array}{l}\text { Substance } \\
\text { abuse }\end{array}$} \\
\hline $\begin{array}{l}\text { Lau et al., } \\
2011\end{array}$ & $\begin{array}{l}54 \text { parent } \\
(41.8,7.2) \\
\text { and child } \\
(8.4,2.0) \\
\text { dyads }\end{array}$ & $\begin{array}{l}100 \text { Chinese } \\
\text { Americans }\end{array}$ & $\begin{array}{l}\text { Time } \\
\text { living in } \\
\text { U.S.: } 2 \\
\text { months-12 } \\
\text { years (for } \\
\text { parents) }\end{array}$ & $\begin{array}{l}\text { Medium } \\
\text { acculturation }^{c}\end{array}$ & Waitlist & Yes & $\begin{array}{l}\text { Behavior } \\
\text { problems }\end{array}$ & Medium \\
\hline \multirow{2}{*}{\multicolumn{9}{|c|}{$\begin{array}{l}\text { Note. AsA = Asian Americans; AfA = African Americans; }{ }^{`}=\text { Values are calculated by the researchers }(\mathrm{N} \text { of people in the ethnic } \\
\text { group/Total N of participants); blank cell indicates data were not obtained or element is not applicable; SDs are not included if they }\end{array}$}} \\
\hline & & & & & & & & \\
\hline \multicolumn{9}{|c|}{ acculturation level was measured by Asian Values Scale (AVS-R; Kim \& Hong, 2004). ${ }^{\mathrm{b}}$ AsA were not included in Study 2.} \\
\hline \multicolumn{9}{|c|}{${ }^{c}$ Acculturation level was measured by Stephenson Multigroup Acculturation Scale (SMAS; Stephenson, 2000). ${ }^{\mathrm{d}}$ AfA mixed race } \\
\hline
\end{tabular}




\section{Figure 1}

Flow diagram for screening and inclusion
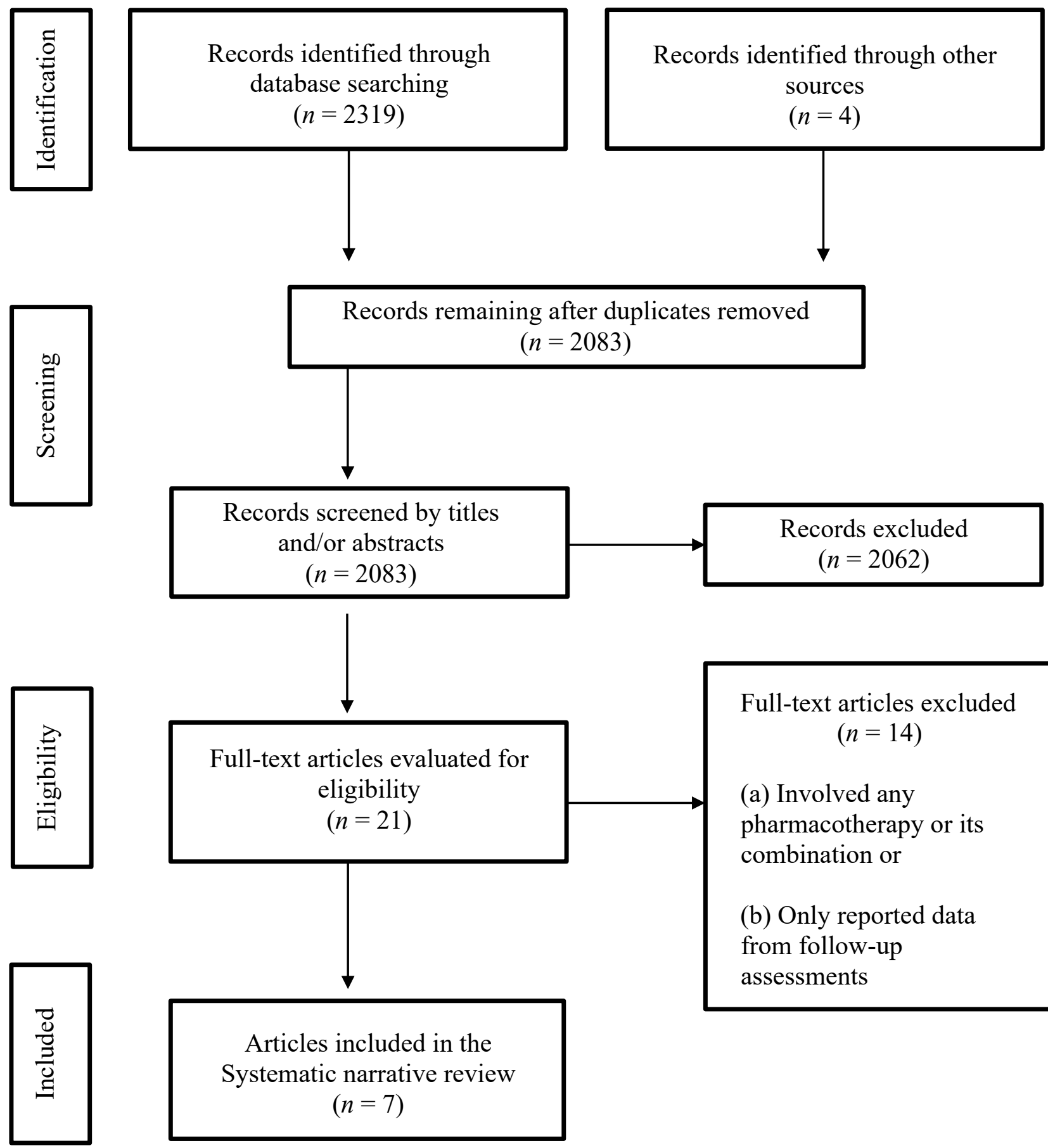

Full-text articles excluded

$$
(n=14)
$$

(a) Involved any pharmacotherapy or its combination or

(b) Only reported data from follow-up assessments

Note. One of the seven articles included two studies (Stice et al., 2014), thus there were a total of eight studies in this review. 